\title{
The Importance of Proximal RCA Stenosis is Associated with Severe OSAS Patients
}

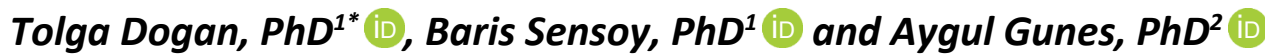 \\ ${ }^{1}$ Department of Cardiology, Bursa Yuksek Ihtisas Education and Research Hospital, Turkey \\ ${ }^{2}$ Department of Neurology, Bursa Yuksek Ihtisas Education and Research Hospital, Turkey
}

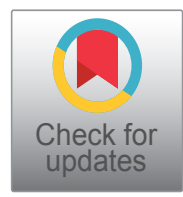

*Corresponding author: Tolga Dogan, Department of Cardiology, Province of Bursa, Bursa Yuksek Ihtisas Education and Research Hospital, District of Yildirim, Neighbourhood of Mimar Sinan, Emniyet Street, 16310, Turkey, Tel: +905055772716, Fax: 0224-366-44-34

\begin{abstract}
Purpose: The aim of this study was to evaluate the relationship of regional selectivity in coronary arteries with OSAS severity using the Syntax (SX) score in OSAS (Obstructive sleep apnea syndrome) patients.

Methods: This retrospective study included 68 OSAS patients with coronary artery disease (CAD) who were followed up at Cardiology and Neurology Outpatient Clinic in our hospital between January 2019 and October 2020. Patients with previously diagnosed OSAS were separated into 2 groups as mild-moderate OSAS group (Group I) and severe OSAS group (Group II). In both groups, coronary angiography was examined using the SX score.

Results: There was no significant difference between the groups in respect of age, gender, smoking status, hyperlipidemia, hypertension and diabetes mellitus. The SX score of the severe OSAS group was significantly higher than that of the mild/moderate group $(14 \pm 8$ vs. 8.3 $\pm 3 p<0.01$ ). RCA proximal critical lesion involvement was significantly higher in the severe group patients $(p<0.01)$.

Conclusion: The results of the current study showed that severe OSAS patients are at higher risk of advanced cardiovascular disease with a higher SX score. Evaluation of right ventricular functions and right coronary artery could be more important insevere OSAS patients.
\end{abstract}

\section{Keywords}

OSAS, Syntax score, Rright coronary artery

\section{Introduction}

Obstructive sleep apnea syndrome (OSAS) is a common disorder that manifests clinically as irregular snoring and sleep rhythm disorders. The apnea- hypopnea index (AHI) is the number of apnea and hypopnea events per hour of sleep documented during polysomnography. $\mathrm{AHI}$ is used as the main criterion to determine and classify the severity of the disesae. OSAS is defined as AHI $\geq 5$. It is often accompanied by diseases such as hypertension, arrhythmia, chronic pulmonary hypertension, type 2 diabetes mellitus, stroke and coronary artery disease (CAD) [1-3]. In many studies, OSAS is accepted as a traditional risk factor for CAD [4-8]. In patients with OSAS, oxygen saturation in the blood decreases as a result of shortness of breath caused by recurrent upper respiratory tract constriction or obstruction during sleep. This causes a series of pathophysiological changes that result in inflammation, endothelial dysfunction, increased oxidative stress, and activation of the sympathetic system, and ultimately these may cause the onset of cardiovascular disease [9-11]. However, even in some studies, the relationship between cardiovascular outcomes and OSAS remains unclear $[12,13]$.

The syntax (SX) score is an anatomic scoring system, which is used to score the severity of CAD according to the complexity, location and functional importance of the coronary lesion. A second use feature of this score is to determine between percutaneous coronary intervention $(\mathrm{PCl})$ and coronary artery bypass grafting in preference for revascularization [14].

In a study with stable CAD using the SX score, it was shown that there is a linear relationship between moderate-to-advanced OSAS disease and the severity

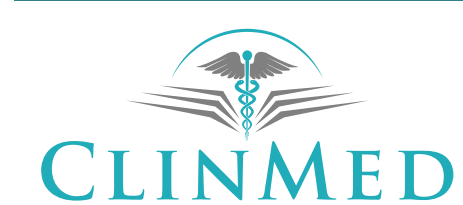

INTERNATIONAL LIBRARY
Citation: Dogan T, Sensoy B, Gunes A (2021) The Importance of Proximal RCA Stenosis is Associated with Severe OSAS Patients. J Sleep Disord Manag 7:036. doi.org/10.23937/2572-4053.1510036 Accepted: October 23, 2021: Published: October 25, 2021

Copyright: (C) 2021 Dogan T, et al. This is an open-access article distributed under the terms of the Creative Commons Attribution License, which permits unrestricted use, distribution, and reproduction in any medium, provided the original author and source are credited. 
Patients received cardiology and neurology outpatient clinics between January 2019 and

October $2020(\mathrm{n}=1535)$

Patients did not have an angiogram or poor qualty

$$
(n=1412)
$$

68 patients were included in the study for evaluation

Figure 1: The study flowchart.

of CAD [15]. While the relationship between severe OSAS and CAD was found in previous studies, the characteristics of the artery segments have not been evaluated.

The aim of this study was to evaluate the relationship of regional selectivity in coronary arteries with OSAS severity using the SX score in OSAS patients.

\section{Patients and Methods}

\section{Patients}

This retrospective study included 1535 OSAS patients in the Cardiology and Neurology Outpatient Clinic in our hospital between January 2019 and October 2020. The study flowchart was shown in Figure 1. Finally, 68 eligible patients were prospectively included in the analysis. The study protocol was approved by the Bursa Yuksek Ihtisas Education and Research Hospital Clinical Research Ethics Committee in accordance with the Declaration of Helsinki and Good Clinical Practice
Guidelines and written informed consent was obtained from all participants (protocol number:2011-KAEK-25 2020/09-06).

The SX score was used for angiographic evaluations of the participants. Exclusion criteria were defined as the presence of rheumatic heart disease, cardiomyopathy, pregnancy, or the use of continuous positive airway pressure (CPAP).

\section{Methods}

Data related to age, gender, smoking status (current, ever, non-smokers), history of hypertension, hypercholesterolemia and diabetes mellitus were collected and recorded from the hospital database.

Polysomnography: In our study, Compumedics brand 58-channel E-Series polysomnography device was used. At least 6 hours of sleep recordings and video images of the participants were recorded. Based on the $\mathrm{AHI}$, the severity of OSAS is classified as mild-moderate 
Table 1: Baseline demographic findings, proximal segmental involvement of coronary arteries and SX scores of both groups.

\begin{tabular}{|c|c|c|c|}
\hline & $\begin{array}{l}\text { Group I } \\
\text { (Mild/Moderate OSAS) } \\
(n=32)\end{array}$ & $\begin{array}{l}\text { Group II } \\
\text { (Severe OSAS) } \\
(n=36)\end{array}$ & $P$ value \\
\hline Age (years) & $57.1 \pm 7$ & $60.2 \pm 8$ & 0.110 \\
\hline Gender (F/M) & $14 / 18$ & $14 / 22$ & 0.684 \\
\hline Hypertension n (\%) & $18(56)$ & $24(67)$ & 0.264 \\
\hline History of Smoking n (\%) & $23(71)$ & $24(67)$ & 0.855 \\
\hline BMI & $26.89 \pm 3.39$ & $24.29 \pm 4.41$ & 0.35 \\
\hline Hyperlipidemia n (\%) & $13(41)$ & $17(48)$ & 0.29 \\
\hline Diabetes Mellitus n (\%) & $10(31)$ & $16(44)$ & 0.193 \\
\hline Syntax Score & $8.3 \pm 3.4$ & $14 \pm 8.3$ & 0.001 \\
\hline \multicolumn{4}{|c|}{ Proximal segmental involvement $\mathrm{n}(\%)$} \\
\hline LAD & $8(25)$ & $10(28)$ & 0.80 \\
\hline $\mathrm{Cx}$ & $3(9)$ & $10(25)$ & 0.17 \\
\hline RCA & $4(13)$ & $14(39)$ & 0.01 \\
\hline
\end{tabular}

BMI: Body Mass İndex

with $\mathrm{AHI}$ 5-30 and severe with $\geq 30 \mathrm{AHI}$ events per hour. Sleep levels and $\mathrm{AHI}$ were manually assessed by certified sleep specialists according to the American Academy of Sleep Medicine criteria [16]. Patients with previously diagnosed OSAS were separated into 2 groups as the mild-moderate OSAS group (Group I) and the severe OSAS group (Group II).

In both groups, coronary angiography was examined using the SX score.

Coronary atherosclerosis and syntax score: Coronary angiographs of all patients were examined by an expert cardiologist using the SX score [In this study Siemens Artis Zee (Siemens AG, Wittelsbachherplatz 2, DE80333Muenchen, Germany) device was used]. Diameter $\geq 1.5 \mathrm{~mm}$ and diameter stenosis of intraluminal lesion $\geq 50 \%$ of all vessels were scored. A lesion causing $>50 \%$ narrowing in the lumen diameter was considered critical. The localization of vascular lesions was determined and recorded. The SX score was calculated using the SYNTAX Score calculator (http://syntaxscore. $\mathrm{com} /$ ). SX score of $\leq 22$ are classified as low risk, those between 23 and 32 as intermediate risk, and those with $\geq 33$ as high risk [17].

\section{Statistical Analysis}

Data obtained in the study were analyzed statistically using SPSS vn. 21.0 software (IBM Inc., Statistical Package for Social Sciences, USA). Descriptive statistics were reported as mean \pm standard deviation values for continuous variables with normal distribution, median and $25^{\text {th }}-75^{\text {th }}$ percentile values for continuous variables without normal distribution, and as frequency ( $\mathrm{n}$ ) and percentage for categorical variables. Group comparisons of continuous variables were made using the Student's t-test and Mann Whitney U-test. Comparisons of categorical variables and evaluation of segment lesions (segmental regional selectivity) with OSAS levels were evaluated with the $\chi^{2}$ test. A value of $p<0.05$ was accepted asstatistically significant in all analyses.

\section{Results}

The 68 participants comprised $36(53 \%)$ in the severe OSAS group and $32(47 \%)$ in the mild-moderate OSAS group. The mean age of the study population was $58 \pm$ 8 years. The baseline characteristics of both groups are shown in Table 1. There was no significant difference between the groups in respect of age, gender, smoking status, hyperlipidemia, BMI (body mass index), hypertension and diabetes mellitus. The mean SX score of the severe OSAS group was significantly higher than that of the mild/moderate group ( $14 \pm 8$ vs. 8.3 $\pm 3, p<0.01)$. In terms of coronary segmental regional selectivity, only the right coronary artery (RCA) proximal critical lesion involvement was significantly higher in Group II than in Group I. Other coronary artery proximal segment lesions did not differ significantly between the groups ( $p>0.05$ ) (Figure 2 ).

\section{Discussion}

The results of this study demonstrated that the SX score and rate of RCA proximal lesion were significantly higher in severe OSAS patients. The difference of this study from previous studies is to investigate whether there is any relationship between severe OSAS and segmental coronary artery disease. Therefore the importance of this study is that it gives clues about the relationship between severe OSAS and coronary artery segments.

The SX score is an angiographic grading procedure to evaluate the complexity of coronary artery disease and The SX score provides optimal revascularization strategies for patients with complex coronary artery disease, and high scores are associated with increasing cardiac mortality and major adverse cardiac events 


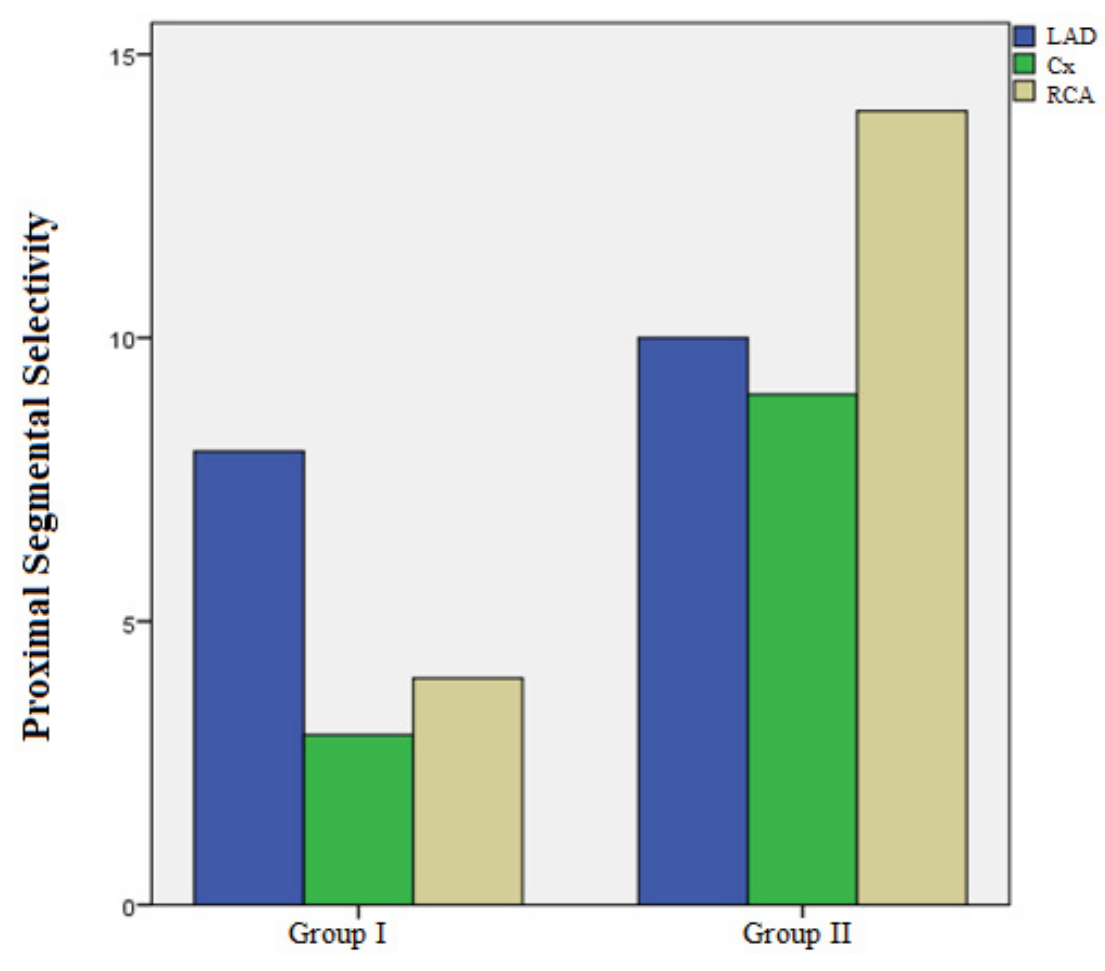

Severity of The OSAS

Figure 2: Proximal segmental severity of RCA, LAD and Cx in both groups.

[14]. In a study by Zhang, et al. the SX score was seen to significantly increase with exacerbation of respiratory sleep disorders in OSAS patients and consequently the severity of coronary artery lesions increased [18].

In an another study conducted on patients with acute coronary syndrome, more chronic total occlusion lesions were detected in OSAS patients compared to patients without OSAS [19]. It has been reported that multivessel disease and coronary artery calcification are more common in OSAS patients [20-22]. In our study we investigated whether there is a specific relationship between SX score and OSAS patients and coronary segments. As a result of the analyzes, we detected proximal RCA lesion more frequently in severe OSAS patients.

The major possible mechanisms include oxidative stress, an abnormal inflammatory response, insulin resistance, sympathetic activation, dyslipidemia, vascular endothelial injury and endothelial dysfunction [23-27]. The mechanisms of endothelial dysfunction in obstructive sleep apnea are: (I) fast reoxygenation and intermittent hypoxia contribute to oxidative stress, thereby increasing sympathetic activity, and inflammation, which lead to endothelial dysfunction (II) Intrathoracic pressure fluctuations create an increased transmural pressure gradient on the vessel walls, and this process causes endothelial dysfunction by causing oscillatory SS [13]. In addition, intrathoracic pressure fluctuations due to the obstructive airway increase venous return and right ventricle (RV) volume overload and RV remodelling [28]. The reason for the severe stenosis in the RCA proximal segment, which was found high in our study, may be due to RV volume overload, especially. Early evaluation of proximal RCA may be considered in OSAS patients with evidence of right ventricular load and $\mathrm{RV}$ remodeling.

A limitation of this study was that the effect of OSAS on remodeling of the right ventricle was not evaluated with echocardiographic data, and IVUS could have been a more valuable method of evaluating endothelial functions. Further studies are needed to investigate the mechanism of these data.

\section{Conclusion}

The results of this study showed that severe OSAS patients are at higher risk of advanced cardiovascular disease with a higher SX score. Therefore, evaluation of right ventricular functions and the right coronary artery can be considered to be more important in severe OSAS patients.

\section{Contributorship}

All of the authors contributed planning, conduct, and reporting of the work. All contributors are responsible for the overall content as guarantors.

\section{Funding}

No funding.

\section{Competing Interests}

All of the authors have no conflict of interest. 


\section{Authors Declaration}

The study was conducted with the approval of the Scientific Research Investigation Commission of Bursa Yuksek Ihtisas Educationand Research Hospital (Ethical approval number: 2011-KAEK-25 2020/09-01 date: 02.09.2020).

\section{References}

1. Buccheri A, Chinè F, Fratto G, Manzon L (2017) Rapid maxillary expansion in obstructive sleep apnea in young patients: Cardio-Respiratory monitoring. J Clin Pediatr Dent 41: 312-316.

2. Ben Halima A, Aouadi S, Bejjar D, Laroussi L, Boukhris M, et al. (2018) Hypertension and atrial fibrillation: What is the prevalence of obstructive sleep apnea syndrome? Tunis Med 96: 187-192.

3. Salmina D, Ogna A, Wuerzner G, Heinzer R, Ogna VF (2019) Hypertension artérielle et syndrome des apnées obstructives du sommeil: état des connaissances [Arterial hypertension and obstructive sleep apnea syndrome: State of knowledge]. Rev Med Suisse 15: 1620-1624.

4. Jiang YQ, Xue JS, Xu J, Zhou ZX, Ji YL (2017) Efficacy of continuous positive airway pressure treatment in treating obstructive sleep apnea hypopnea syndrome associated with carotid arteriosclerosis. Exp Ther Med 14: 6176-6182.

5. Arous F, Boivin JM, Chaouat A, Rumeau C, Jankowski R, et al. (2017) Awareness of obstructive sleep apnea-hypopnea syndrome among the general population of the Lorraine region of France. Eur Ann Otorhinolaryngol Head Neck Dis 134: 303-308.

6. Luo H, Scholp A, Jiang JJ (2017) The finite element simulation of the upper airway of patients with moderate and severe obstructive sleep apnea hypopnea syndrome. Biomed Res Int 2017: 7058519.

7. Karimzadeh F, Nami M, Boostani R (2017) Sleep microstructure dynamics and neurocognitive performance in obstructive sleep apnea syndrome patients. J Integr Neurosci 16: 127-142.

8. Alvarez-Sabín J, Romero O, Delgado P, Quintana M, Santamarina E, et al. (2018) Obstructive sleep apnea and silent cerebral infarction in hypertensive individuals. J Sleep Res 27: 232-239.

9. Alonso-Fernandez A, Garcia-Rio F, Arias MA, Hernanz A, de la Peña M, et al. (2009) Effects of CPAP on oxidative stress and nitrate efficiency in sleep apnoea: A randomised trial. Thorax 64: 581-586.

10. Cowie MR (2017) Sleep apnea: State of the art. Trends Cardiovasc Med 27: 280-289.

11. Javaheri S, Barbe F, Campos-Rodriguez F, Dempsey JA, Khayat R, et al. (2017) Sleep Apnea: Types, mechanisms, and clinical cardiovascular consequences. J Am Coll Cardiol 69: 841-858.

12. Barbe F, Duran-Cantolla J, Sanchez-de-la-Torre $M$, Martinez-Alonso M, Carmona C, et al. (2012) Effect of continuous positive airway pressure on the incidence of hypertension and cardiovascular events in nonsleepy patients with obstructive sleep apnea: A randomized controlled trial. JAMA 307: 2161-2168.

13. Kohler M, Stradling JR (2010) Mechanisms of vascular damage in obstructive sleep apnea. Nat Rev Cardiol 7: 677-685.
14. Sianos G, Morel MA, Kappetein AP, Morice MC, Colombo A, et al. (2005) The SYNTAX Score: An angiographic tool grading the complexity of coronary artery disease. Eurolntervention 1: 219-227.

15. Kochergin NA, Kochergina AM, Ganyukov VI, Barbarash OL (2020) [Predictors of coronary plaque vulnerability in patients with stable coronary artery disease]. Kardiologiia 60: 20-26.

16. Patil SP, Ayappa IA, Caples SM, Kimoff RJ, Patel SR, et al. (2019) Treatment of adult obstructive sleep apnea with positive airway pressure: An American Academy of Sleep Medicine Clinical Practice Guideline. J Clin Sleep Med 15: 335-343.

17. Farooq V, Brugaletta S, Serruys PW (2011) The SYNTAX score and SYNTAX-based clinical risk scores. Semin Thorac Cardiovasc Surg 23: 99-105.

18. Zhang T, Zhang C, Chen RX, Shao B, Liu GA (2018) Correlation between coronary artery lesion quantitative score and OSAHS and relative risk factors. Eur Rev Med Pharmacol Sci 22: 1415-1420.

19. Zeng Y, Yang S, Wang X, Fan J, Nie S, et al. (2019) Prognostic impact of residual SYNTAX score in patients with obstructive sleep apnea and acute coronary syndrome: A prospective cohort study. Respir Res 20: 43.

20. Abinader EG, Peled N, Sharif D, Lavie P (1994) STsegment depression during obstructive sleep apnea. Am J Cardiol 73: 727.

21. Javadi HR, Jalilolghadr S, Yazdi Z, Rezaie Majd Z (2014) Correlation between obstructive sleep apnea syndrome and cardiac disease severity. Cardiovasc Psychiatry Neurol 2014: 631380.

22. Sorajja D, Gami AS, Somers VK, Behrenbeck TR, GarciaTouchard A, et al. (2008) Independent association between obstructive sleep apnea and subclinical coronary artery disease. Chest 133: 927-933.

23. Trenchea M, Deleanu O, Suţa M, Arghir OC (2013) Smoking, snoring and obstructive sleep apnea. Pneumologia 62: 5255.

24. Davies PF (2009) Hemodynamic shear stress and the endothelium in cardiovascular pathophysiology. Nat Clin Pract Cardiovasc Med 6: 16-26.

25. Tan S, Liu X, Xu Y, Luo L, Zhou S, et al. (2017) Serum highdensity lipoprotein correlates with serum apolipoprotein $M$ and $\mathrm{A} 5$ in obstructive sleep apnea hypopnea syndrome. Sleep Breath 21: 37-44.

26. Karkinski D, Georgievski O, Dzekova-Vidimliski P, Milenkovic T, Dokic D (2017) Obstructive sleep apnea and lipid abnormalities. Open Access Maced J Med Sci 5: 1922.

27. Guan J, Yi H, Zou J, Meng L, Tang X, et al. (2016) Distinct severity stages of obstructive sleep apnoea are correlated with unique dyslipidaemia: Large-scale observational study. Thorax 71: 347-355.

28. Li J, Lin X, Li H, Lu C, Li R, et al. (2020) Right ventricular diastolic dysfunction in patients with obstructive sleep apnea syndrome. Echocardiography 37: 317-322. 